\title{
Effect of surface stiffness on the neural control of stretch- shortening cycle movements
}

\author{
G. Márquez,' L. Morenilla, ${ }^{2}$ W. Taube $^{3}$ and M. Fernández-del-Olmo ${ }^{2}$ \\ I Departamento de Ciencias de la Actividad Física y del Deporte, Facultad de Ciencias de la Actividad Física y del Deporte, UCAM, \\ Universidad Católica San Antonio, Murcia, Spain \\ 2 Leaming and Human Movement Control Group, Facultad de Ciencias de la Actividad Física y del Deporte, University of A Coruña, \\ A Coruña, Spain \\ 3 Department of Medicine, Movement and Sports Science, University of Fribourg, Fribourg, Switzerland
}

Correspondence: G. Márquez, $\mathrm{PhD}$, Faculty of Sport Sciences, Catholic University of Murcia, Avda. de Los Jerónimos S/N 30107 Murcia, Spain E-mail: gmarquez@ucam.edu

\begin{abstract}
Aim: It is accepted that leg stiffness $\left(K_{\text {leg }}\right)$ increases when surface stiffness decreases, and vice versa. However, little is known how the central nervous system fulfils this task. To understand the effect of surface stiffness on the neural control of stretch-shortening cycle movements, this study aimed to compare modulation of spinal and corticospinal excitability at distinct phases after ground contact during two-legged hopping when changing from solid to elastic ground.

Methods: Motor-evoked potentials (MEPs) induced by transcranial magnetic stimulation (TMS) and H-reflexes were elicited at the time of the short (SLR)-, medium (MLR)- and long (LLR)-latency responses of the soleus muscle (SOL) during two-legged hopping on different stiffness surfaces, elastic and stiff.

Results: Soleus H-reflexes during two-legged hopping on the elastic surface were lower at SLR and larger at LLR than on the stiff surface $(P<0.05$ for both comparisons). SOL MEP size was higher at the time of SLR during hopping on the elastic surface than on the stiff surface $(P<0.05)$ although the background EMG was similar.

Conclusion: It is argued that this phase-specific adaptation in spinal reflex excitability is functionally relevant to adjust leg stiffness to optimally exploit the properties of the elastic surface. Thus, the increased corticospinal excitability on the elastic surface may reflect a more supraspinal control of the ankle muscles to compensate the decrease in reflexive stiffness at the beginning of touchdown and/or counteract the higher postural challenges associated with the elastic surface.

Keywords H-reflex, leg stiffness, stretch-shortening cycle, transcranial magnetic stimulation.
\end{abstract}

The neural control of stretch-shortening cycle (SSC) movements such as drop jumps, two-legged hopping, or running is highly complex incorporating feedforward (pre-programmed) and feedback (reflex) control (Taube et al. 2012a). Thus, multiple hierarchical levels of the central nervous system (CNS) have to closely interact to adjust neural control task (Leukel et al. 2008a) and context specifically (Leukel et al. 2008a, 2012) taking into account the biomechanical constraints of the human body. The CNS is therefore challenged to optimally exploit the capacity of the tendomuscular system to store kinetic energy during 
the eccentric phase (touchdown), which can subsequently be released in the concentric phase (push-off) without exposing the body to the risk of being overloaded. It was previously demonstrated that the CNS can adjust the recoil properties of the tendomuscular system in various ways at different times of the SSC movement; for instance Arampatzis et al. (2001) showed that the level of pre-activation (muscular activity before touchdown) is related to the stiffness of the lower extremity. Furthermore, the reflex-induced activity shortly after touchdown can be modulated. Several studies observed reduced H-reflex activity with increasing drop height (Leukel et al. 2008a,b). Interestingly, the reduction in the amplitude of the H-reflex with increasing drop height was positively correlated with a decrease of the lower leg stiffness (Taube et al. 2012b). Furthermore, when subjects were asked to land instead of rebounding from the ground, the $\mathrm{H}$ reflex excitability was largely reduced (Leukel et al. 2008b). Thus, there is good evidence that adaptation of both pre-programmed voluntary activity and feedback-mediated reflex activity contribute to stiffness control in SSC movements.

For everyday locomotion, neural control of leg stiffness may be particularly important when changes in the support surface occur. Based on kinematic data, it is for instance known that the movement pattern executed during jumping on solid ground, where the legs first are flexed and extended subsequently, is changed to a reversed pattern on an elastic surface (Moritz \& Farley 2005). The question is how the CNS can adapt the initial motor command to allow this drastic change. Moritz \& Farley (2005) observed reduced stretch reflex responses but increased overall muscular activity when rebounding from soft elastic surfaces. The authors interpreted their findings in the way that the CNS had to compensate for the loss of the stretch reflex contribution when aiming to maintain the normal centre of mass dynamics. The observation that repeated jumping on an elastic surface leads to after effects when subjects are tested on solid ground afterwards (Márquez et al. 2010) strengthens the hypothesis that the central motor command is adapted surface specifically. However, to date, it is not clear how the CNS fulfils this task. This study therefore aimed to compare spinal and corticospinal excitability when changing from solid to elastic ground. Previous experiments on solid ground have shown that in both hopping and drop jumping the H-reflex excitability was low at take-off, still low in the flight phase, but increased before touchdown. During the initial ground contact at the time of the short-latency response (SLR), the H-reflex was facilitated but subsequently decreased towards take-off (Moritani et al. 1990, Dyhre-Poulsen et al. 1991, Voigt et al. 1998, Taube et al. 2008). In contrast, motor-evoked potentials (MEPs) elicited by transcranial magnetic stimulation (TMS) were low shortly after ground contact but increased towards the push-off phase in drop jumps. The authors therefore concluded that the first EMG peak after touchdown (SLR) strongly relies on Ia-afferent feedback while the corticospinal facilitation at push-off points to a phase-specific facilitation of transcortical loops at this later time. This study aimed to clarify how these particular patterns of spinal and corticospinal excitability are modified on an elastic surface. Based on the above-mentioned observations of Moritz \& Farley (2005) and the finding that the motor cortex is involved at the SLR during two-legged hopping (Zuur et al. 2010), we hypothesized that spinal reflex excitability should be decreased on elastic ground at the time of the SLR but might be - at least partly - compensated by supraspinal contribution.

\section{Methods}

\section{Participants}

Sixteen healthy subjects $(173.1 \mathrm{~cm}, \quad 72.3 \mathrm{~kg}$, 25.3 years; all the participants were right leg dominant) without record of neurological or orthopaedic disorder participated in this study. Ten subjects (two female) were recruited for the main experiment, and six subjects (one female) for the complementary experiment. Before testing, all subjects gave written consent about the experimental procedure. The experiments were run in accordance with the Declaration of Helsinki and approved by the local ethics committee of the University of A Coruña. The study conforms with Persson (2013).

\section{General experimental procedure}

Main experiment. Each experimental session started with a standardized warm-up protocol. At the end of the warm-up, subjects were instructed to perform two-legged hopping at $2.2 \mathrm{~Hz}$ on two surfaces that differed with respect to their stiffness. The solid surface consisted of a force plate with a stiffness of approx. $35000 \mathrm{kN} \mathrm{m}^{-1}$, whereas the elastic (soft) surface comprised a spring floor mounted over the force plate with a much lower stiffness $\left(60 \mathrm{kN} \mathrm{m}^{-1}\right.$; Fig. 1). These two different experimental conditions were carried out in a counterbalanced order in the same session. During two-legged hopping, TMS was applied over the contralateral primary motor cortex (M1) of the leg area using a double-cone coil secured to the head through a custom-made helmet. H-reflex of the soleus (SOL) was obtained by electrical stimulation of the posterior tibial nerve using two different 


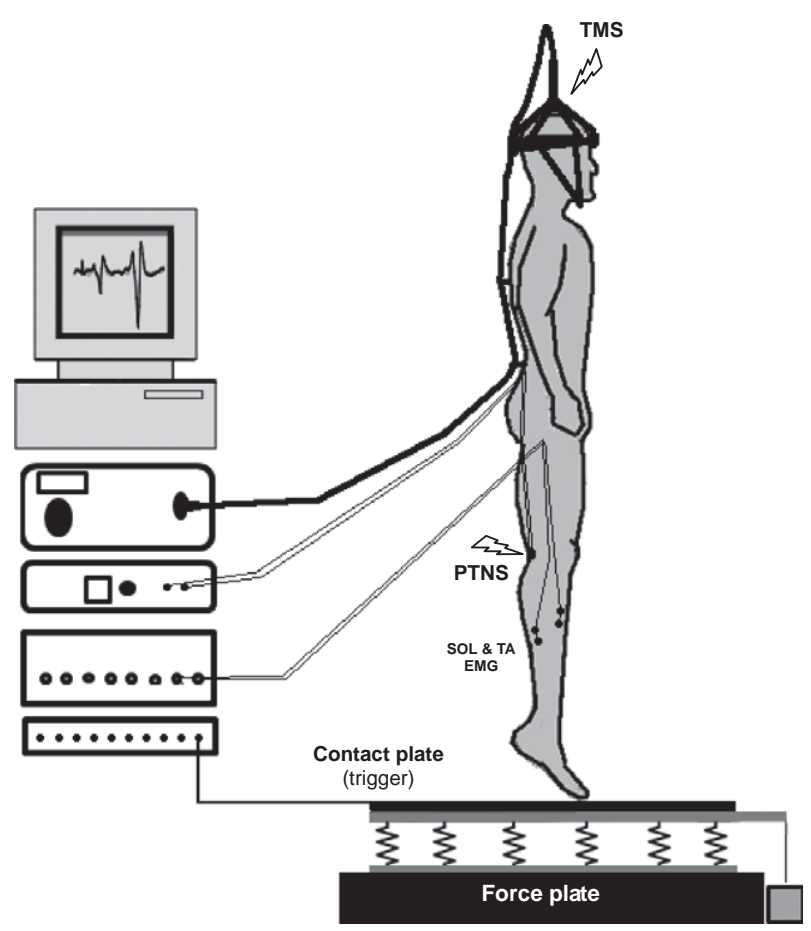

Figure I Illustration of the experimental set-up. TMS was applied to the contralateral primary motor cortex (M1) of the leg area using a double-cone coil secured to the head through a custommade helmet (90\% RMT intensity). $\mathrm{H}$-reflexes and M-waves of the SOL were obtained by electrical stimulation (intensity was adjusted to elicit $\mathrm{M}$-waves of 20 and $50 \%$ of $\mathrm{M}_{\max }$, respectively) of the posterior tibial nerve (PTNS). All of the recordings were triggered by means of a contact conductive platform located on top of the elastic and the stiff surface. intensities, 20 and $50 \%$ of the $M_{\max }$ (low and high stimulation). MEPs, H-reflexes and background EMG (bEMG) of the soleus of the right leg were recorded during two-legged hopping at distinct time intervals after ground contact: at the time of the short-latency response (SLR, $45 \mathrm{~ms}$ ), the medium-latency response (MLR, 70) and the long-latency response (LLR, $120 \mathrm{~ms})$. The latencies and durations of the abovementioned intervals were previously reported (Petersen et al. 1998, Grey et al. 2001, Kawashima et al. 2004, Leukel et al. 2008a,b, Taube et al. 2008).

At the beginning of the experiment, subjects were asked to perform four bouts of two-legged hopping at $2.2 \mathrm{~Hz}$. The duration of each bout was $50 \mathrm{~s}$, and 110 hops were collected. In every bout, subjects were tested in three different randomized conditions: hopping without stimulation (only the bEMG was recorded), hopping with electrical stimulation using two stimulation intensities (the $\mathrm{M}$-wave was adjusted to 20 and $50 \%$ of $M_{\max }$ ) and hopping with TMS. Subjects were instructed to rest for $2-3 \mathrm{~min}$ between trials. Electrical and magnetic stimulation were triggered so that the peak of the H-reflex and the MEP, respectively, coincided with the peaks of the SLR, MLR or LLR (methodological approach in accordance with Taube et al. 2008). For each stimulation point (SLR, MLR and LLR), ten trials at low and high electrical stimulation intensity and 20 trials with TMS were recorded. Moreover, 320 trials were collected without stimulation so that the corresponding bEMG activity could be determined. After subjects were tested on one surface, they rested for $10 \mathrm{~min}$ and then repeated the same protocol on the other surface. Figure 2 displays the main protocol.

Control blocks during standing position. In seven of the ten subjects, SOL H-reflex recruitment curves and SOL MEPs were recorded during standing position just before and after the first hopping bout, and after the second hopping bout that was performed on the other surface.

Hopping instructions. Subjects were instructed to perform two-legged hopping at $2.2 \mathrm{~Hz}$ with their arms held akimbo. A digital metronome provided the designated frequency of $2.2 \mathrm{~Hz}$, which was previously shown to be the preferred hopping frequency for humans (Farley et al. 1991, Hobara et al. 2007). Subjects were required to look straight ahead to a fixed target on the wall that served as a visual reference.

Hopping surfaces. Subjects were instructed to hop on a $92 \times 92 \mathrm{~cm}$ force platform (stiffness \pm $35000 \mathrm{kN} \mathrm{m}^{-1}$; see Ferris \& Farley 1997) and on a spring floor mounted on the force platform. The spring floor consisted of a modified gymnastic springboard that was firmly secured to the force plate by webbing straps. The linear stiffness of the elastic surfaces was $60 \mathrm{kN} \mathrm{m}^{-1}$. The stiffness of the elastic surface was evaluated by static load tests in which weights (up to $2000 \mathrm{~N}$ ) were placed on the centre of the spring floor, and the displacement of the surface 
START

Figure 2 Schematic representation of the main experimental protocol. In this experiment, ten subjects were tested to compare corticospinal and spinal contributions during hopping on two different stiffness surfaces, an elastic surface and a stiff surface. In seven subjects, SOL MEPs elicited by suprathreshold TMS (1.2 rMT) and SOL H-M recruitment curves were also obtained while subjects stood at rest just before the beginning of the hopping task, during the $10 \mathrm{~min}$ recovery period and at the end of the session (control blocks).

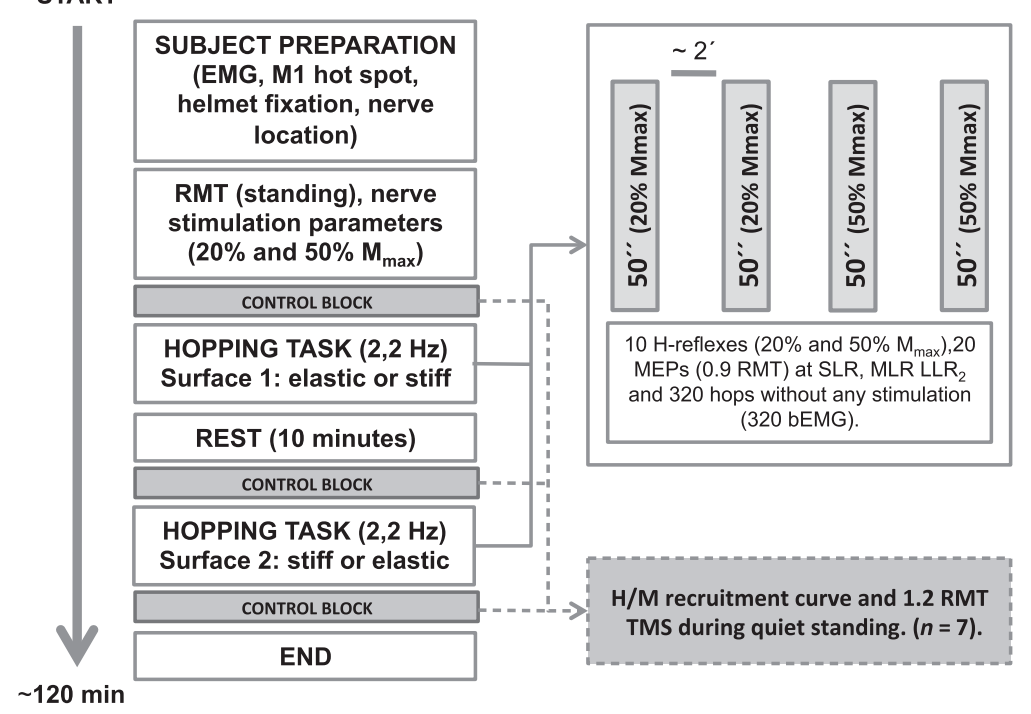

was measured (in line with Ferris \& Farley 1997). The linear regression between force and displacement was high $\left(r^{2}=0.99\right)$. On top of both surfaces, a contact conductive platform was placed to trigger the start and the end of ground contact.

Mechanical recordings. Vertical ground reaction forces (GRF) were recorded using a force platform (Kistler model: 9290AD) and stored on a PC for further analysis. A linear encoder (Musclelab Bosco System; Ergotest Technology, MA.GI.CA. srl, Roma, Italy) was attached to the ground floor and to the top of the spring floor to assess the elastic surface displacement.

EMG recordings. EMG signals were recorded from the soleus (SOL) and tibialis anterior (TA) muscles of the right leg. $\mathrm{Ag}-\mathrm{AgCl}$ surface electrodes (diameter: $10 \mathrm{~mm}$, centre to centre distance: $3 \mathrm{~cm}$ ) were filled with electrode jelly and attached to the skin in line with the presumed direction of the underlying muscle fibres. The reference electrode was placed on the lateral malleolus of the fibula. Electrodes and cables were secured with an adhesive tape and elastic mesh to prevent possible artefacts caused by the highly dynamic movement. EMG signals were amplified (SOL: $\times 300$ or $\times 500$ depending on the size of the $M_{\max }$; TA $\left.\times 1000\right)$, bandpass-filtered $(30-1000 \mathrm{~Hz})$ and sampled at $2 \mathrm{kHz}$ with a Digitimer D360 amplifier (Digitimer, Welwyn Garden City, UK) connected to an AD board (Power1401; Cambridge Electronic Design, Cambridge, UK) for further analysis.

H-reflex. The soleus H-reflex was elicited by stimulation of the posterior tibial nerve using a $1.5 \mathrm{~cm}^{2}$ cathode located in the popliteal fossa and a $5 \times 5 \mathrm{~cm}$ anode fixed just underneath the patella. The optimum site for nerve stimulation was located using a handheld electrode. It was ensured that stimulation evoked no response in the TA muscle. The stimulus was a square-wave pulse of $1 \mathrm{~ms}$ duration applied by a constant current electrical stimulator (DS7AH; Digitimer). The cathode was fixed with rigid tape. First, an H-reflex recruitment curve was recorded during upright stance. Afterwards, stimulation intensity was adjusted to elicit $\mathrm{M}$-waves of 20 and $50 \%$ of the $M_{\max }$. This ensured the activation of the same portion of the motor pool in each subject resulting in an $\mathrm{H}$ reflex being on the ascending slope of the H-reflex recruitment curve (Crone et al. 1990). In the main experiment, we have also used a stimulation intensity to elicit $\mathrm{M}$-waves of $50 \%$ of $M_{\max }$ to obtain a sensitive measure for changes in the stimulation intensity (Pinniger et al. 2001, Taube et al. 2008).

TMS. Transcranial magnetic stimulation was applied over the left motor cortex using a double-cone coil connected to a Magstim 200 (Magstim, Dyfed, UK). The stimulus waveform was monophasic and had a pulse width of $200 \mu$ s. For determining the best stimulation position in each subject, the coil was situated $\pm 0.5 \mathrm{~cm}$ posterior to the vertex and over the midline and was moved anterior and left from the vertex while MEP size of SOL and TA were monitored on an oscilloscope. While the subject rested on a comfortable chair, the hot spot was located and it was marked on the scalp with a felt pen. Then, the coil was fixed firmly to the head to secure the optimal position for eliciting MEPs in the SOL muscle during stance with minimal stimulator output. Resting motor threshold (rMT) of the SOL muscle was defined as the 
stimulator intensity at which three of five consecutive MEPs reached $150 \mu \mathrm{V}$ (Kujirai et al. 1993). According to Taube et al. (2008), the threshold was set to $150 \mu \mathrm{V}$ because most of the subjects showed relatively high tonic muscular activation during stance $( \pm 100 \mu \mathrm{V})$. Stimulation intensity during jumps was adjusted to 0.9 rMT (in accordance with Taube et al. (2008) as in some subjects, an intensity equal or higher than the rMT disrupted their hopping movement pattern (G. Márquez, L. Morenilla, W. Taube, M. Fernández-del-Olmo, unpublished observations).

To ensure a constant stimulation site throughout the hopping task, the coil was fixed to a customdesigned helmet, which provided a flexible adjustment to the head size. The helmet was built following recommendations mentioned by previous studies that assessed corticospinal contribution by TMS during whole-body movements such as hopping and jumping (Taube et al. 2008, Zuur et al. 2010). The coil could be moved relative to the helmet by an aluminium system that allowed highly flexible handling. To minimize any forces acting on the helmet, the cable of the coil was separately fixed to the back of the subject. The helmet and the cable were also supported by elastic bands attached to the ceiling. This set-up reduced the weight of the coil and the cable so that the equipment did not disturb subjects' jumping behaviour.

Complementary experiment. In a separate session with six different subjects, H-reflex recruitment curves at each stimulation point (SLR, MLR and LLR) were recorded during two-legged hopping on the solid and the elastic surface. Participants were asked to perform 12 bouts of $40 \mathrm{~s}$ hopping on each surface. Subjects rested for $2 \mathrm{~min}$ between bouts and $10 \mathrm{~min}$ between surfaces. In each bout, electrical stimulation of the posterior tibial nerve was randomly applied at SLR, MLR and LLR. The intensity was adjusted in steps of $10 \%$ of the maximal $\mathrm{M}$ response recorded during upright stance, beginning from the H-reflex threshold until $110 \%$ of $M_{\max }$ (in accordance with Zehr 2002).

\section{Data analysis}

Mechanical parameters. To determine the mechanical behaviour during two-legged hopping, GRF were recorded. Vertical acceleration was computed from the GRF to obtain the vertical velocity and displacement of the centre of mass [CoM] using the double integration method (Cavagna 1975).

Vertical displacement of the CoM and the peak GRF $\left(F_{\text {peak }}\right)$ were used to compute leg stiffness $\left(K_{\text {leg }}\right)$ and total stiffness $\left(K_{\text {total }}\right) . K_{\text {leg }}$ represents the average stiffness of the overall musculoskeletal system during the ground contact phase, and $K_{\text {total }}$ is defined as the combination of the stiffness of the surface and the stiffness of the legs (Farley et al. 1991). In this way, $K_{\text {total }}$ was computed as the ratio between the peak GRF and the maximum CoM displacement during contact phase: $K_{\text {total }}=F_{\text {peak }} / \Delta y_{\text {total }}$. $K_{\text {leg }}$ was obtained by taking the ratio of the peak ground reaction force $\left(F_{\text {peak }}\right)$ and the maximum compression of the legs $(\Delta \mathrm{L})$ : $K_{\text {leg }}=F_{\text {peak }} / \Delta \mathrm{L}$, where $\Delta \mathrm{L}$ was determined by subtracting the surface displacement $\left(\Delta \mathrm{y}_{\text {surf }}\right)$ from the total CoM displacement $\left(\Delta \mathrm{y}_{\text {total }}\right): \quad \Delta \mathrm{L}=\Delta \mathrm{y}_{\text {total }}-\Delta \mathrm{y}_{\text {surf }}$. Contact time (CT) and flight time (FT) were also computed from the ground reaction force signal. Then, hopping frequency $(\omega)$ was calculated using the following equation: $\omega=1 /[\mathrm{CT}+\mathrm{FT}]$.

Neurophysiological parameters. Based on previous reports about the latencies of the SLR, MLR and LLR during SSC movements (Taube et al. 2008), we selected three time windows in which the muscular activity was assessed. The MEP, H-reflex and bEMG amplitudes were determined by calculating peak to peak values over a $20 \mathrm{~ms}$ time window around the SLR (i.e. 35-55 ms after ground contact), MLR (i.e. 60-80 ms) and LLR (i.e. $110-130 \mathrm{~ms}$ ). For all subjects, the main peak of activation for the respective phase (SLR, MLR and LLR) fell into this time window. Then, H-reflex and MEP responses were normalized using the method proposed by Taube et al. (2008), where the bEMG (mean of all trials without stimulation) was subtracted from the H-reflex (mean of all trials obtained with peripheral nerve stimulation) or from the MEPs (mean of all trials obtained with TMS) and then normalized to the corresponding M-wave $50 \%$ \{formula: [(HR or MEP - bEMG)/Mwave $\left._{50 \%}\right]^{* 100\}}$. In case of the TA muscle, MEP amplitudes were expressed as a percentage of the corresponding background EMG activity analysed in the respective time interval as we did not obtain an $\mathrm{M}$-wave in this muscle that could serve as a reference. Additionally, we analysed the bEMG amplitudes during the pre-activation phase in a $50-\mathrm{ms}$ window prior to touchdown. Co-contraction of antagonistic muscle pairs was also assessed for the respective time intervals (SLR, MLR and LLR), and it was expressed in the following way: TA bEMG/SOL bEMG.

To control for systematic confounding effects such as fatigue, movement of the coil or the stimulation electrodes, we compared $H_{\max } / M_{\max }$ ratios obtained from the SOL H-reflex recruitment curves and the average of ten consecutive SOL MEPs elicited with a suprathreshold TMS intensity of 1.2 rMT during upright stance before, at 10-min rest period (between surfaces) and after the last hopping bout.

In the complementary experiment, H-reflex and Mwave sizes were measured as peak to peak amplitudes. 
An average of ten responses for each stimulation intensity and each stimulation point (SLR, MLR and LLR) was taken into account for the analysis. The $H_{\text {max }} / M_{\max }$ ratio was used because it represents the best sensitive measure to assess changes in spinal reflex circuitries (Zehr 2002).

\section{Statistics}

For the main experiment, two-way repeated measures ANOVAs were performed with surface (elastic or stiff) and stimulation point (SLR, MLR and LLR) as factors for the following variables: SOL and TA bEMG, SOL and TA MEPs, SOL H-reflexes obtained with intensities to elicit $\mathrm{M}$-waves of 20 and $50 \%$ of $M_{\max }$, respectively, and co-contraction level. In the complementary experiment, the same analysis was performed for the $H_{\max } / M_{\max }$ ratios. To analyse the control measures, a one-way repeated measures ANOVA (time trial as a factor: before, $10 \mathrm{~min}$ rest and after) was performed for the SOL $H_{\max } / M_{\max }$ ratio and for the SOL MEP size. To compare the mechanical behaviour during hopping on the two different stiffness surfaces, a Student's paired $t$-test was performed for the following parameters: $K_{\text {leg }}, K_{\text {total }}, F_{\text {peak }}, \Delta L$, Ay total, hopping frequency $(\omega)$, contact and flight time (CT and FT). All data were normally distributed, and statistical significance was set at $P \leq 0.05$. Post hoc analysis was performed using paired comparisons with Bonferroni correction. SPSS 15.0 software (SPSS, Chicago, IL, USA) was used for statistical analysis. Data are presented as group mean values \pm standard error of the mean (SEM).

\section{Results}

\section{Main experiment}

Mechanical behaviour. The mechanical behaviour of hopping on both surfaces (stiff and elastic) is presented in Table 1. Analysis revealed a significant higher $K_{\operatorname{leg}}$ during hopping on the elastic surface than on the stiff surface $(t=7.42, \quad P<0.001)$. This increase in leg stiffness is due to a significant lower leg compression $(\Delta \mathrm{L})(t=27.90, P<0.001)$, as a small, although significant, decrease in $F$ peak during hopping on the elastic surface was found $(t=3.06$, $P=0.016)$. No significant differences were evident for $K_{\text {total }}, \mathrm{Ay}_{\text {total }}$, frequency, contact and flight time.

Background EMG recordings. For the SOL background EMG activity, the ANOVA showed a main effect of stimulation point $(F=4.57, P=0.025$; Fig. $3 a)$. Analysis revealed lower bEMG amplitude at LLR compared with SLR $(t=0.53, P=0.049)$. TA bEMG
Table I Mechanical recordings measured during hopping on the stiff and elastic surface

\begin{tabular}{lcc}
\hline & \multicolumn{1}{l}{ Stiff } & \multicolumn{1}{l}{ Elastic } \\
\hline Frequency $(\mathrm{Hz})$ & $2.20 \pm 0.020$ & $2.18 \pm 0.035$ \\
Contact time $(\mathrm{s})$ & $0.24 \pm 0.011$ & $0.25 \pm 0.007$ \\
Flight time $(\mathrm{s})$ & $0.21 \pm 0.012$ & $0.21 \pm 0.011$ \\
$F_{\text {peak }}(\mathrm{kN})$ & $2.60 \pm 0.157$ & $2.37 \pm 0.107^{*}$ \\
$K_{\text {total }}\left(\mathrm{kN} \mathrm{m}^{-1}\right)$ & $20.34 \pm 1.303$ & $17.42 \pm 0.893$ \\
$K_{\text {leg }}\left(\mathrm{kN} \mathrm{m}^{-1}\right)$ & $20.34 \pm 1.303$ & $27.16 \pm 1.737^{* *}$ \\
$\Delta \mathrm{L}(\mathrm{m})$ & $0.13 \pm 0.001$ & $0.09 \pm 0.002^{* *}$ \\
$\Delta \mathrm{y}_{\text {total }}(\mathrm{m})$ & $0.13 \pm 0.001$ & $0.14 \pm 0.002$ \\
\hline
\end{tabular}

$* P \leq 0.05 ; * P \leq 0.0001$.

activity did not show any significant differences (Fig. 3b). Similarly, muscular activity in the pre-activation phase was not different between the stiff and the elastic surface for both the soleus and the tibialis muscle. Regarding to the co-contraction, the analysis did not show statistical differences between jumps on the stiff and the elastic surface.

Normalized MEP size of SOL and TA. ANOVA-MR revealed a main effect of surface $(F=6.08, P=0.033)$ and stimulation point $(F=8.42, P=0.002)$ and a surface*stimulation point interaction $(F=3.42$, $P=0.049)$. Normalized SOL MEP size was statistically higher during hopping on the elastic surface than on the stiff surface at the SLR $(t=4.01, P=0.002)$, but not in the $\operatorname{MLR}(t=1.95, P=0.080)$ nor $\operatorname{LLR}(t=0.51$, $P=0.624)$. Moreover, normalized SOL MEP size was higher at LLR compared with SLR and MLR $(P<0.05$ for both comparisons; Fig. 3c) when subjects jump on both surfaces, stiff and elastic. In contrast, ANOVA did not show significant main effects for TA MEP size; however, a tendency towards a significant surface*stimulation point interaction was found $(F=3.29, P=0.060$; paired $t$-test: SLR $t=1.93, P=0.080$; MLR $t=1.32$, $P=0.220$; LLR $t=0.02, t=0.980$; Fig. $3 \mathrm{~d}$ ).

Raw data of one subject hopping on the elastic and on the stiff surface are displayed in Figure 4. The left side shows the increase in SOL MEP amplitudes at the time of the SLR, MLR and LLR as soon as the subject performs hopping on the elastic surface.

Peripheral nerve stimulation. Electrical stimulation with low and high intensity revealed a main effect of stimulation point (low intensity $F=9.45$, $P=0.001$; high intensity $F=17.60, P<0.001)$ and a significant surface*stimulation point interaction (low intensity $F=3.78, P=0.040$; high intensity $F=3.75$,

$P=0.041$; Fig. 5a,b). The Post hoc analysis demonstrated that changing from the stiff to the elastic 

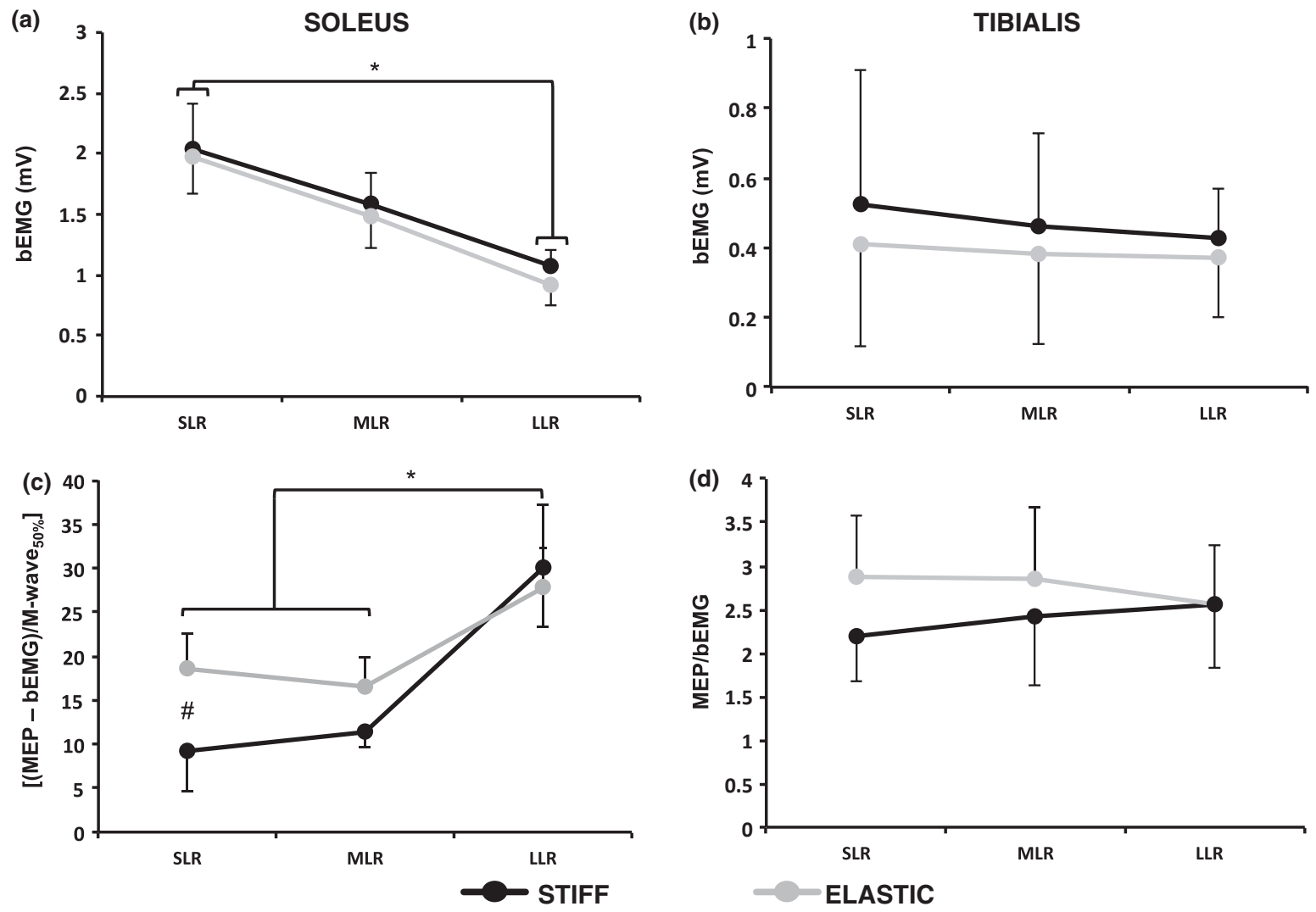

Figure 3 Background EMG and normalized MEP size of the SOL and TA muscles at different time intervals (SLR, MLR and LLR) during two-legged hopping on the elastic (grey lines) and stiff surface (black lines). No differences were found in the SOL and TA bEMG during hopping on both surfaces $(\mathrm{a}-\mathrm{b})$; subjects showed higher SOL MEPs size during hopping on the elastic surface than on the stiff surface at the time of SLR (c). A clear tendency to a higher TA MEP size was found in the SLR time interval during hopping on the elastic surface (d). (\#) Differences between surfaces. (*) Difference between stimulation points.

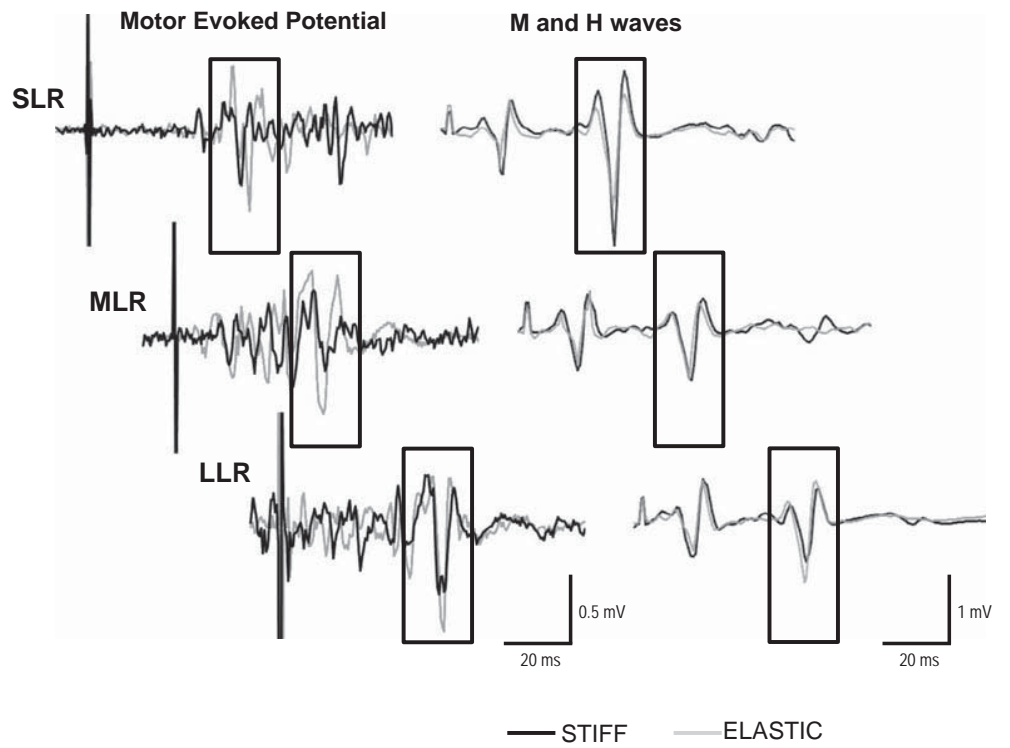

Figure 4 Ensemble average of the SOL MEP (left panel) and H-reflex (right panel) obtained at SLR, MLR and LLR during hopping on the elastic (grey traces) and on the stiff surface (black traces) in a single subject. It can be seen that MEPs increased from SLR to MLR and LLR. Furthermore, MEP amplitudes were larger on the elastic than on the stiff surface at the time of SLR. In contrast, H-reflex amplitudes were largest at the time of the SLR and were subsequently reduced towards push-off (LLR). Switching from the stiff to the elastic surface resulted in lower $\mathrm{H}$-reflexes at the time of the SLR and increased Hreflex amplitudes at LLR. (\#) Differences between surfaces.

surface significantly reduced H-reflex size at the time of the SLR (low-intensity stimulation $t=2.92$, $P=0.008$; high-intensity stimulation $t=2.25, P=$
0.035 ) but increased H-reflex activity at LLR (lowintensity stimulation $t=1.90, P=0.045$; high-intensity stimulation $t=2.19, P=0.039)$. No surface-specific 

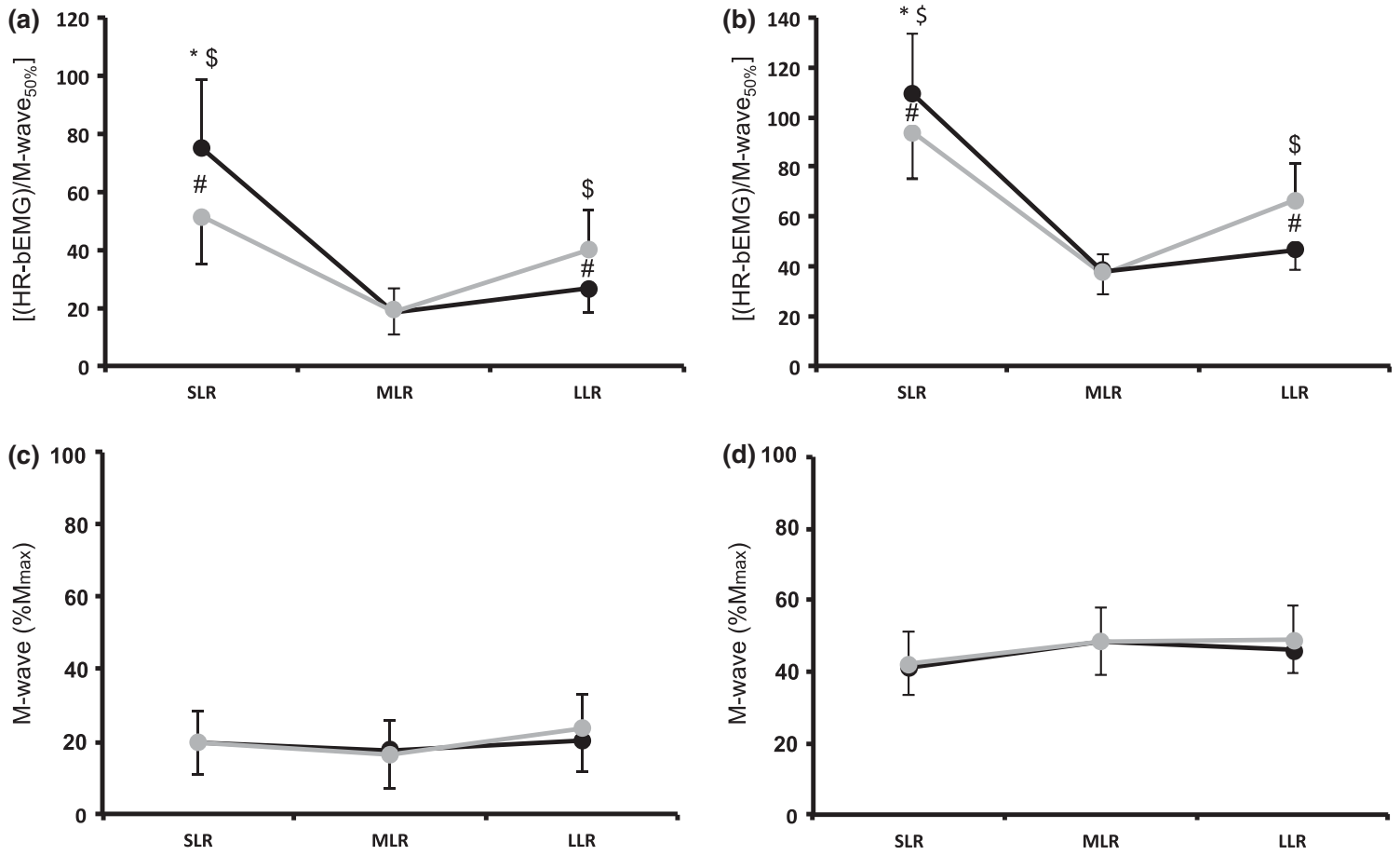

Figure 5 Results from peripheral nerve stimulation. H-reflexes were significantly higher during hopping on the stiff surface (black line) than on the elastic surface (grey line) at SLR. However, a reversed pattern was obvious at LLR while H-reflexes at MLR showed no changes. This pattern was evident for both low (a) and high (b) stimulation intensity. There were no differences in the M-wave amplitudes obtained at SLR, MLR and LLR neither with low (c) and high (d) stimulation intensities nor between surface conditions. (\#) Differences between surfaces. $\left(^{*}\right)$ Difference between SLR with respect to MLR and LLR during hopping on the stiff surface. (\$) Difference between SLR and LLR with respect to MLR during hopping on the elastic surface.

differences occurred at MLR. Post hoc analysis also revealed significantly higher $\mathrm{H}$-reflexes at SLR compared with MLR and LLR during hopping on the stiff surface $(P<0.05$ for both stimulation intensities $)$ and significantly higher H-reflexes at SLR and LLR compared with MLR during hopping on the elastic surface $(P<0.05$ for both stimulation intensities). M-waves were comparable across surface conditions and stimulation points (Fig. 5c,d). Raw data of one subject are displayed in Figure 4 on the right side. It can be seen that when changing from the stiff to the elastic surface, the H-reflex is reduced at SLR but increased at LLR.

Control blocks during standing position. No differences were found in the SOL $H_{\max } / M_{\max }$ ratio or in the SOL MEP size during the control blocks performed before, at the 10-min recovery period and after the last hopping bout (Table 2).

Complementary experiment. The mean group data \pm SEM (i) and individual values (ii) of the $H_{\max } / M_{\max }$ ratios obtained at the SLR, MLR and LLR during hopping on both surfaces confirm and complement the above-mentioned H-reflex results (Fig. 6). The ANOVA-RM showed a significant main effect for the stimulation point $(F=4.47, P=0.041)$ and a significant surface* stimulation point interaction $(F=25.98$, $P<0.001)$. The post hoc analysis demonstrated that the $H_{\max } / M_{\max }$ ratios were significantly higher during hopping on the stiff surface than on the elastic surface at $\operatorname{SLR}(t=5.16, P=0.004)$. In contrast, changing the stiffness of the surface had different effects on the $H / M$ ratios at LLR resulting in higher ratios on the elastic surface $(t=3.76, P=0.013)$.

\section{Discussion}

The present study investigated how humans adjusted their leg stiffness during two-legged hopping to accommodate changes in surface stiffness and in what way the descending drive and the processing of afferent feedback were modified to accomplish this mechanical adaptation. The current results obtained during hopping confirm previous observations made in drop jumps (Taube et al. 2008), showing a time-specific modulation of spinal and corticospinal excitability during the stretch-shortening phase. Furthermore and even more importantly, the present study highlights how corticospinal excitability is modulated in response to changes of the surface 
Table 2 SOL MEP amplitude (in $\mathrm{mV}$ ) and $H_{\max } / M_{\max }$ ratios during control blocks. No differences were found in any parameter

\begin{tabular}{llll}
\hline & Before & 10-min rest & After \\
\hline$H_{\max } / M_{\max }$ & $0.55( \pm 0.06)$ & $0.51( \pm 0.07)$ & $0.49( \pm 0.07)$ \\
Soleus MEPs & $0.51( \pm 0.14)$ & $0.53( \pm 0.18)$ & $0.47( \pm 0.12)$ \\
\hline
\end{tabular}

properties. SOL H-reflex during hopping on the elastic surface was lower at SLR and larger at LLR than on the stiff surface. SOL MEP size was higher during hopping on the elastic surface than on the stiff one. Our research therefore revealed for the first time how processing of Ia-afferent discharge and corticospinal excitability of the SOL muscle are affected by changes in the surface stiffness during a whole-body locomotor task such as two-legged hopping.

Mechanical adaptations due to changes in surface stiffness. In the present study, subjects increased their leg stiffness about $33.5 \%$ and reduced lower leg compression about $-31 \%$ during hopping on the elastic surface. These adjustments resulted in similar CoM motions during hopping on both surfaces as no differences were found in $\mathrm{Ay}_{\text {total }}$, FT and CT. Those findings are in line with previous studies that reported increased leg stiffness when surface stiffness is decreased (Farley et al. 1998, Ferris \& Farley 1997, Ferris et al. 1998, Ferris et al. 1999; Moritz \& Farley 2004). Consequently, adjustments made in $K_{\text {leg }}$ resulted in similar total stiffness of the series combination of the leg and the surface during hopping on the two different floor surfaces (in accordance to Farley et al. 1998), indicating that adaptations in $K_{\text {leg }}$ may have served to compensate the loss of surface stiffness. In accordance with Moritz \& Farley (2004), the constant $K_{\text {total }}$ allowed the ground contact time and aerial time to remain nearly the same, making it possible for the CoM mechanics to be remarkably similar at a given hopping frequency regardless of the surface stiffness.

Spinal adaptations in response to changes in the stiffness surface. Previous SSC studies revealed that $\mathrm{H}-$ reflex excitability is modulated in a phase-dependent fashion: H-reflex was increased before touchdown, remained facilitated during the stance phase and decreased before push-off (Dyhre-Poulsen et al. 1991, Voigt et al. 1998, Taube et al. 2008). Based on these observations, it was suggested that the first EMG peak after touchdown (SLR) strongly relies on Ia-afferent feedback (Dyhre-Poulsen et al. 1991, Taube et al. 2008). This was recently confirmed by experiments, where the landing surface was either lowered or lifted while subjects were airborne during hopping (Zuur et al. 2010). It was hypothesized that if the activity at the time of the SLR remained unchanged by altering the height of the landing surface, the muscular activity had to be pre-programmed and cannot result from a stretch reflex. However, this was not the case. Instead, muscular activity of the SLR was shifted forward when the platform was lifted and backward when it was lowered, indicating strong contribution of stretch reflexes at this phase.

In accordance with these previous observations, results from the present study have shown a similar phase-dependent modulation of the soleus H-reflex
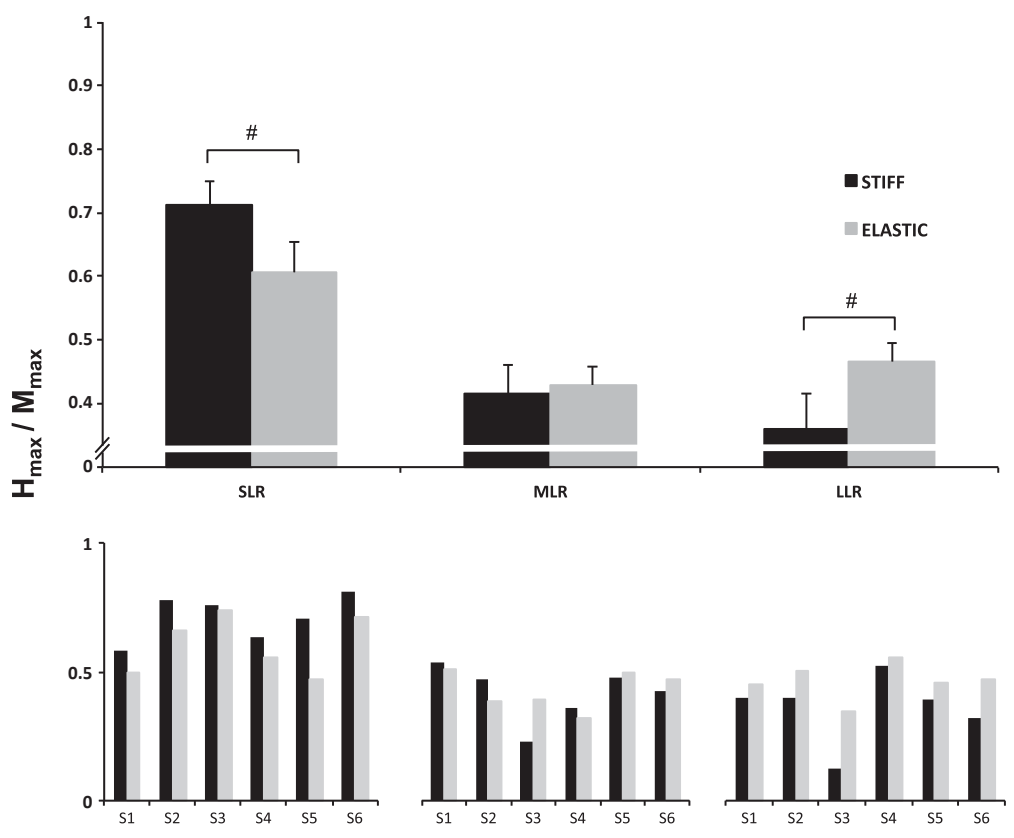

Figure 6 Group mean data \pm SEM (top) and individual values (down) of the ratio $H_{\max } / M_{\max }$ obtained at SLR, MLR and LLR during hopping on the elastic (grey bars) and stiff (black bars) surfaces. This control experiment confirms the previous observation displayed in Figure 5. (\#) Differences between surfaces. 
during hopping on the stiff surface: the H-reflex amplitude was high at SLR and thereafter progressively decreased at MLR and LLR, suggesting that muscular activity at those later time points (MLR and LLR) was not as much depending on Ia-afferent input than at the time of the SLR (Taube et al. 2008). Interestingly, SOL $\mathrm{H}$-reflexes at the time of the SLR were lower when subjects were required to hop on the elastic surface than on the stiff surface. These changes in Ia-afferent transmission in the early stance phase may explain earlier findings of Moritz \& Farley (2005) reporting reduced EMG activity at SLR on elastic ground. It may be speculated that on elastic floor, changes at the spinal level such as an increase in pre-synaptic inhibition may have reduced stretch-induced activity at the time of the SLR. It is obvious that such adaptations at the spinal level would have to be pre-programmed as time constraints prevent online correction. In this respect, recent findings indicated that subjects can anticipate the requirements of different surfaces and adapt their motor control in a feedforward manner (Moritz \& Farley 2004). Furthermore, several SSC studies have provided indication that gating of spinal reflex circuits at the time of the SLR is pre-programmed, probably by pre-synaptic inhibition (Leukel et al. 2008a,b, 2012, Taube et al. 2012a).

The switch to the elastic surface had also an effect on the H-reflex response at the time of the LLR. Independent of how we measured the H-reflex (M-waves adjusted to 20 or $50 \%$ of $M_{\max } ; H / M$ ratios), increased excitability was observed at LLR. The latency of the LLR response corresponded to the latency where the subject surface system was maximally compressed $( \pm 120 \mathrm{~ms})$. It may therefore be assumed that enhanced reflex contributions may have been important to resist potential further muscle lengthening by increasing the stiffness (Nichols \& Houk 1973, Nichols \& Houk 1976) at the time of maximal compression.

Corticospinal adaptations in response to changes in the stiffness surface. The present study revealed higher SOL MEP size and a tendency for TA MEPs at the time of the SLR during two-legged hopping on the elastic surface compared with the stiff one. Although it is difficult to know whether this change is cortical in origin, it seems to be independent from those adjustments at the spinal level. Therefore, the higher corticospinal excitability of SOL and TA on the elastic surface may reflect a higher supraspinal control of these muscles that might have helped to adjust leg stiffness to the new surface compliance. Alternatively or additionally, the higher corticospinal excitability during hopping on the elastic surface may be related to the increased postural demand that subjects face when rebounding from the more unstable surface. It is important to remark that the mean surface displacement was $5 \mathrm{~cm}$, meaning that subjects had to take this mechanical instability into account. Increased corticospinal excitability is known to go along with unstable stance conditions like standing tiptoe or on a free-swinging platform (Lavoie et al. 1995, Solopova et al. 2003). In this line, Tokuno et al. (2009) have also reported higher cortical excitability during normal stance than in a supported stance condition. Furthermore, there is strong evidence from electrophysiological recordings in both rabbits and cats that the firing of cortical neurones is strongly related to the postural correction (Beloozerova et al. 2003, 2005). In this regard, the increased corticospinal excitability on the elastic surface may at least partly result from the need to account for the increased postural challenge.

Methodological considerations. A potential methodological concern with our protocol is that during hopping, high forces act over the whole body, which could affect the coil position during the experiment. In this sense, previous studies using high-speed cameras and frameless stereotaxic neuro-navigation systems have shown that the movement of the coil was maintained within $2 \mathrm{~mm}$ of the target (Taube et al. 2008, Zuur et al. 2010). In fact, in the current experiment, MEP modulation during hopping on the stiff surface resembled MEP modulation observed during drop jumps (Taube et al. 2008). Moreover, our control measures performed at the beginning of the experiment, during the $10 \mathrm{~min}$ rest period and after the last hopping bout showed no differences in the SOL MEPs proposing a constant coil position during the entire experiment.

Further methodological issues related to the H-reflex measurements during hopping could be the difference in joint angles between the conditions (Gerilovsky et al. 1989, Leukel et al. 2008a). As the design of our study resulted in differential kinematics on the stiff and the elastic surface, altered joint angles may have caused a shift of the stimulation electrode relative to the underlying nerve fibres (see Zehr 2002). To account for this limitation, we conducted a complementary experiment to ensure comparable stimulation intensities between conditions where H-M recruitment curves were recorded at each stimulation point (SLR, MLR and LLR). This experiment produced similar results to those observed in the main protocol.

\section{Functional consideration and conclusion}

In summary, adaptations in both the H-reflex and the MEP were observed when subjects changed from a 
stiff to a more compliant surface. From a functional point of view, these adaptations are highly plausible. Excitability of spinal reflexes was reduced at the initial contact but increased later on where the highest compression of the body took place. Thus, reflexes were most likely adapted to provide adequate phase-specific tendomuscular stiffness for each surface.

In contrast, corticospinal excitability was increased on the elastic surface at the time of SLR. In part, this may be due to the loss of efficacy of spinal reflex responses on elastic ground so that supraspinal centres had to compensate the loss of reflex contribution (argumentation in line with Moritz \& Farley 2005). However, the higher postural challenges on the elastic surfaces may have also demanded stronger supraspinal involvement. This might also explain the tendency for a MEP increase in the TA at the time of the SLR, where the lower leg had to be stabilized on the elastic floor.

\section{Conflict of interest}

There is no conflict of interest.

\section{References}

Arampatzis, A., Brüggemann, G.P. \& Klapsing, G.M. 2001. Leg stiffness and mechanical energetic processes during jumping on a sprung surface. Med Sci Sports Exerc 33, 923-931.

Beloozerova, I.N., Sirota, M.G., Swadlow, H.A., Orlovsky, G.N., Popova, L.B. \& Deliagina, T.G. 2003. Activity of different classes of neurons of the motor cortex during postural corrections. J Neurosci 23, 7844-7853.

Beloozerova, I.N., Sirota, M.G., Orlovsky, G.N. \& Deliagina, T.G. 2005. Activity of pyramidal tract neurons in the cat during postural corrections. J Neurophysiol 93, 18311844.

Cavagna, G.A. 1975. Force platforms as ergometers. J Appl Physiol 39, 174-179.

Crone, C., Hultborn, H., Mazières, L., Morin, C., Nielsen, J. \& Pierrot-Deseilligny, E. 1990. Sensitivity of monosynaptic test reflexes to facilitation and inhibition as a function of the test reflex size: a study in man and the cat. Exp Brain Res 81, 35-45.

Dyhre-Poulsen, P., Simonsen, E.B. \& Voigt, M. 1991. Dynamic control of muscle stiffness and $\mathrm{H}$ reflex modulation during hopping and jumping in man. J Physiol 437, 287-304.

Farley, C.T., Blickhan, R., Saito, J. \& Taylor, R. 1991. Hopping frequency in humans: a test of how spring set stride frequency in bouncing gaits. J Appl Physiol 71, 21272132.

Farley, C.T., Houdijk, H.H., Van Strien, S.C. \& Louie, M. 1998. Mechanism of leg stiffness adjustment for hopping on surfaces of different stiffnesses. J Appl Physiol 85, 1044-1055.
Ferris, D.P. \& Farley, C.T. 1997. Interaction of leg stiffness and surfaces stiffness during human hopping. J Appl Physiol 82, 15-22.

Ferris, D.P., Louie, M. \& Farley, C.T. 1998. Running in the real world: adjustments in leg stiffness for different locomotion surfaces. Proc Biol Sci 265, 989-994.

Ferris, D.P., Liang, K. \& Farley, C.T. 1999. Runners adjust leg stiffness for their first step on a new running surface. $J$ Biomech 32, 787-794.

Gerilovsky, L., Tsvetinov, P. \& Trenkova, G. 1989. Peripheral effects on the amplitude of monopolar and bipolar H-reflex potentials from the soleus muscle. Exp Brain Res 76, 173-181.

Grey, M.J., Ladouceur, M., Andersen, J.B., Nielsen, J.B. \& Sinkjaer, T. 2001. Group II muscle afferents probably contribute to the medium latency soleus stretch reflex during walking in humans. J Physiol 534, 925-933.

Hobara, H., Kanosue, K. \& Suzuki, S. 2007. Changes in muscle activity with increase in leg stiffness during hopping. Neurosci Lett 418, 55-59.

Kawashima, N., Nakazawa, K., Yamamoto, S.I., Nozaki, D., Akai, M. \& Yano, H. 2004. Stretch reflex excitability of the anti-gravity ankle extensor muscle in elderly humans. Acta Physiol Scand 180, 99-105.

Kujirai, T., Caramia, M.D., Rothwell, J.C., Day, B.L., Thompson, P.D., Ferbert, A., Wroe, S., Asselman, P. \& Marsden, C.D. 1993. Corticocortical inhibition in human motor cortex. J Physiol 471, 501-519.

Lavoie, B.A., Cody, F.W. \& Capaday, C. 1995. Cortical control of human soleus muscle during volitional and postural activities studied using focal magnetic stimulation. Exp Brain Res 103, 97-107.

Leukel, C., Gollhofer, A., Keller, M. \& Taube, W. 2008a. Phase- and task-specific modulation of soleus H-reflexes during drop-jumps and landings. Exp Brain Res 190, 71-79.

Leukel, C., Taube, W., Gruber, M., Hodapp, M. \& Gollhofer, A. 2008b. Influence of falling height on the excitability of the soleus H-reflex during drop-jumps. Acta Physiol (Oxf) 192, 569-576.

Leukel, C., Taube, W., Lorch, M. \& Gollhofer, A. 2012. Changes in predictive motor control in drop-jumps based on uncertainties in task execution. Hum Mov Sci 31, 152160.

Márquez, G., Aguado, X., Alegre, L.M., Lago, A., Acero, R.M. \& Fernández-del-Olmo, M. 2010. The trampoline aftereffect: the motor and sensory modulations associated with jumping on an elastic surface. Exp Brain Res 204, 575-584.

Moritani, T., Oddsson, L. \& Thorstensson, A. 1990. Differences in modulation of the gastrocnemius and soleus H-reflexes during hopping in man. Acta Physiol Scand 138, 575-576.

Moritz, C.T. \& Farley, C.T. 2004. Passive dynamics change leg mechanics for an unexpected surface during human hopping. J Appl Physiol 97, 1313-1322.

Moritz, C.T. \& Farley, C.T. 2005. Human hopping on very soft elastic surfaces: implications for muscle pre-stretch and elastic energy storage in locomotion. J Exp Biol 208, 939-949. 
Nichols, T.R. \& Houk, J.C. 1973. Reflex compensation for variations in the mechanical properties of a muscle. Science 181, 182-184.

Nichols, T.R. \& Houk, J.C. 1976. Improvement in linearity and regulation of stiffness that results from actions of stretch reflex. J Neurophysiol 39, 119-142.

Persson, P.B. 2013. Good Publication Practice in Physiology 2013: revised author guidelines for Acta Physiologica. Acta Physiol (Oxf) 209, 250-253.

Petersen, N., Christensen, L.O., Morita, H., Sinkjaer, T. \& Nielsen, J. 1998. Evidence that a transcortical pathway contributes to stretch reflexes in the tibialis anterior muscle in man. J Physiol 512, 267-276.

Pinniger, G.J., Nordlund, M., Steele, J.R. \& Cresswell, A.G. 2001. H-reflex modulation during passive lengthening and shortening of the human triceps surae. J Physiol 534, 913923.

Solopova, I.A., Kazennikov, O.V., Deniskina, N.B., Levik, Y.S. \& Ivanenko, Y.P. 2003. Postural instability enhances motor responses to transcranial magnetic stimulation in humans. Neurosci Lett 337, 25-28.

Taube, W., Leukel, C., Schubert, M., Gruber, M., Rantalainen, T. \& Gollhofer, A. 2008. Differential modulation of spinal and corticospinal excitability during drop jumps. J Neurophysiol 99, 1243-1252.

Taube, W., Leukel, C. \& Gollhofer, A. 2012a. How neurons make us jump: the neural control of stretch-shortening cycle movements. Exerc Sport Sci Rev 40, 106-115.

Taube, W., Leukel, C., Lauber, B. \& Gollhofer, A. 2012b. The drop height determines neuromuscular adaptations and changes in jump performance in stretch-shortening cycle training. Scand J Med Sci Sports 22, 671-683.

Tokuno, C.D., Taube, W. \& Cresswell, A.G. 2009. An enhanced level of motor cortical excitability during the control of human standing. Acta Physiol (Oxf) 95, 385-395.

Voigt, M., Dyhre-Poulsen, P. \& Simonsen, E.B. 1998. Modulation of short latency stretch reflexes during human hopping. Acta Physiol Scand 163, 181-194.

Zehr, E.P. 2002. Considerations for use of the Hoffmann reflex in exercise studies. Eur J Appl Physiol 86, 455468.

Zuur, A.T., Lundbye-Jensen, J., Leukel, C., Taube, W., Grey, M.J., Gollhofer, A., Nielsen, J.B. \& Gruber, M. 2010. Contribution of afferent feedback and descending drive to human hopping. J Physiol 588(Pt 5), 799-807. 